\title{
Kognitiv-psychoedukative Therapie im Vergleich zu Bibliotherapie bei bipolarer Störung: Eine kontrollierte Gruppentherapiestudie
}

\author{
Gerhard Lenz Andrea Berg Beatrix Breit-Gabauer \\ Sabine Lorenz-Demelbauer Itha Stampfer Martin Aigner \\ Marion Freidl Michael Ossege Markus Schaffer
}

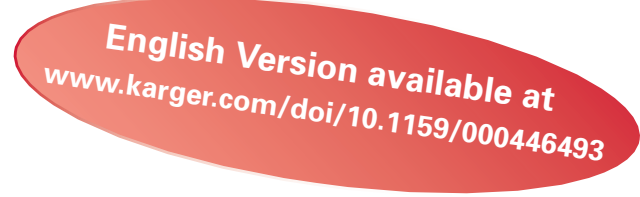

Klinische Abteilung für Sozialpsychiatrie, Universitätsklinik für Psychiatrie und Psychotherapie, AKH Wien, Wien, Österreich

\section{Schlüsselwörter}

Bipolare Störung · Kognitiv-psychoedukative Therapie · Bibliotherapie · Gruppentherapie · Kontrollierte Studie

\section{Zusammenfassung}

Hintergrund: Kognitive Verhaltenstherapie wird als zusätzlich wirksam zur medikamentösen Therapie in der Rückfallprophylaxe bei bipolaren Störungen angesehen. Es gibt bisher allerdings nur wenige Untersuchungen zu Therapien mit deutschsprachigen Therapiemanualen. PatientInnen und Methoden: In einer kontrollierten Gruppentherapiestudie für bipolare PatientInnen wurde die Wirksamkeit der kognitiv-psychoedukativen Therapie (KPT) nach dem Therapiemanual von Schaub et al. [2004] im Vergleich zu einer Bibliotherapie (BT) auf die Rückfallhäufigkeit hin untersucht. Ergebnisse: Insgesamt wurden 100 medizierte PatientInnen über 14 Wochen behandelt, 52 in der KPT-Gruppe und 48 in der BT-Gruppe. 12 Monate nach Beginn der Studie schnitten in der Globalbeurteilung nach der Clinical Global Impression (CGI)Skala die PatientInnen der KPT-Gruppe signifikant besser ab als die der BT-Gruppe. In beiden Gruppen zeigt sich eine signifikante Reduktion der Anzahl der Krankheitsepisoden (in der BT-Gruppe allerdings nur für die manischen Episoden) und der Anzahl und Dauer stationärer Krankenhausaufenthalte. Außerdem konnten in beiden Gruppen positive Auswirkungen auf Krankheitskonzepte und eine Reduktion psychosozialer Beeinträchtigungen gezeigt werden. Schlussfolgerungen: Beide Therapieformen sind wirksam in der Vorbeugung manischer Episoden, während nur die KPT-Gruppe auch einen Einfluss auf die Verhinderung depressiver Episoden sowie insgesamt eine höhere Wirksamkeit zeigte.

(c) 2016 S. Karger GmbH, Freiburg
Keywords

Bipolar Disorder - Cognitive-psychoeducative therapy . Bibliotherapy · Group therapy · Controlled trial

\section{Summary}

Background: There is evidence for the efficacy of cognitive behavioural therapy in addition to pharmacotherapy in patients with bipolar disorder. But until now, there is only a limited number of studies with therapy manuals in the German language. Patients and Methods: The efficacy of cognitive psychoeducative therapy (CPT) according to the manual of Schaub et al. [2004] versus bibliotherapy (BT) on the relapse rates in patients with bipolar disorder was evaluated in a controlled group therapy study. Results: 100 patients on prophylactic medication were treated over 14 weeks: 52 in the CPT group and 48 in the BT group. At the 12-month follow-up after start of treatment, the patients of the CPT group had a significantly better outcome in the Clinical Global Impression (CGI) scale than those in the BT group. There was a significant reduction in the overall number of episodes in both groups (but only for manic episodes in the BT group) and also in the number and duration of hospital admissions in the 12 months after start of treatment compared to the 12 months before. In both groups, there was a positive effect on illness concepts (Linden scale) and a reduction of disability (Sheehan scale). Conclusions: Both kinds of therapy were successful in relapse prevention of manic episodes; the CPT group also showed an inhibitory influence regarding depressive episodes, and CPT was generally more effective. 


\section{Einleitung}

Vulnerabilitäts-Stress-Coping-Modelle [Meyer und Hautzinger, 2004] sehen das zentrale Kernproblem bipolar affektiver Störungen in einer Instabilität oder Dysregulation biologischer Prozesse (z.B. Schlaf-Wach-Zyklus, zirkadiane Rhythmen usw.), die durch kritische Lebensereignisse bzw. Stress (z.B. Konflikte, Arbeitsbelastung, gravierende berufliche oder private Veränderungen usw.) oder Alkohol-/Drogenkonsum getriggert werden können, wobei zur Stabilisierung einerseits medikamentöse Maßnahmen, andererseits psychotherapeutische Interventionen und die Aktivierung individueller Ressourcen beitragen können.

Die medikamentöse Therapie sowohl in akuten Phasen der Erkrankung als auch in der Prophylaxe ist heute unbestritten die Grundlage jeder Behandlung [DGPPN, 2012; Grunze et al., 2013]. Probleme mit der Adhärenz für die Medikation, der hohe Anteil von Krankheitsverläufen ohne Vollremission und der Einfluss psychosozialer Faktoren auf den Verlauf sind Ansatzpunkte für psychoedukative und psychotherapeutische Verfahren als Zusatz zur medikamentösen Therapie. Hier spielen vor allem die kognitive Verhaltenstherapie (KVT), die familienfokussierte Therapie und die interpersonelle und soziale Rhythmustherapie eine wichtige Rolle [Überblicke über Anwendung und Wirksamkeit bei Hautzinger und Meyer, 2007; Schöttle et al., 2011; Schaub und Neubauer, 2013; Reinares et al., 2014].

Ein offenes Forschungsproblem ist die Frage, ob eine spezifische Psychotherapie wirksamer ist als eine reine Psychoedukation bzw. eine stützende Therapie. Ob KVT bei gleicher Behandlungsintensität (als Einzeltherapie) wirksamer ist als stützende Therapie, wurde in der Studie von Meyer und Hautzinger [2012] untersucht: Hier zeigten sich keine Unterschiede zwischen den Gruppen in den Rückfallraten, und es wurde von den Autoren diskutiert, ob vor allem die gemeinsamen Charakteristika wie Information und Stimmungsmonitoring (was sich als förderlich für Selbstmanagement und Selbstregulation herausstellte) die Wirkung psychosozialer Interventionen erklären.

Für die KVT zeigen Meta-Analysen [Szentagotai und David, 2010] eine bessere Wirksamkeit als eine Wartelistenkontrolle, eine kurze Psychoedukation oder «Treatment as Usual» (TAU). Eine kleinere Meta-Analyse von Lynch et al. [2010] stellt diese guten Ergebnisse allerdings infrage. Mittlerweile gibt es seit etwa 10 Jahren auch in deutscher Sprache mehrere Therapieanleitungen bzw. Therapiemanuale zur Psychotherapie [Meyer und Hautzinger, 2004; Schaub et al., 2004] und zur Psychoedukation [Wagner und Bräunig, 2004; Jelley und Elmer, 2005; Erfurth et al., 2005].

Die kognitiv-psychoedukative Therapie (KPT) wurde bereits in einer kontrollierten Studie von Bernhard [2009] an 52 PatientInnen mit bipolarer Störung untersucht. Davon nahmen 24 PatientInnen an einer KPT-Gruppe teil und 27 Personen an einer Wartekontrollgruppe. Nach 1 Jahr zeigte sich im Vergleich zur Kontrollgruppe eine signifikante Reduktion der Krankheitstage sowie der depressiven Symptome. In einer klinischen Verlaufsstudie an 52 PatientInnen mit bipolarer Störung von Schaub et al. [2013] verbesserten sich die Teilnehmer der kognitiv-psychoedukativen
Gruppe ( $\mathrm{n}=24$ ) zwischen dem Beginn und dem Abschluss der Gruppe signifikant im Wissenstest und bezüglich der Schwere der Erkrankung. Über 96\% der Teilnehmer bewerteten das KPT-Gruppenangebot als hilfreich bis sehr hilfreich.

Unter Bibliotherapie (BT) versteht man den Einsatz von Texten zu therapeutischen Zwecken. Sie kann alleine als Selbsthilfeliteratur angewendet oder in Ergänzung zu einer Gruppen- oder Einzelpsychotherapie eingesetzt werden [Überblick: Grahlmann und Linden, 2005]. Die Akzeptanz und Wirksamkeit der BT ist nach diesen Autoren nicht nur über den Inhalt der dargebotenen Information bestimmt, sondern auch durch die psychologischen Prozesse der Einstellungs- und Verhaltensänderungen, die durch die Texte angestoßen werden. Ein weiterer ausführlicher Überblick findet sich bei Niedermayer [2006]. Die Effektivität von BT wird in Meta-Analysen im mittleren Bereich $(\mathrm{d}=0,56-0,84)$ [zitiert bei Herm, 2010] in der Anwendung bei verschiedensten psychischen Störungen (meist unipolare Depressionen) angegeben. Für bipolare Störungen gibt es bisher noch keinen empirischen Wirknachweis für die BT [DGPPN, 2012: S3-Leitlinien].

Für die Kontrollgruppe wurde die BT deshalb gewählt, weil hier die Information und Psychoedukation von einer spezifischen Psychotherapie gut abgrenzbar und vom zeitlichen Umfang (Lesen eines Buches von 250 Seiten verbunden mit 3 90-minütigen Gruppensitzungen) ähnlich aufwendig wie die 14 KPT-Gruppensitzungen erschienen. Wir wollten also in unserer Studie untersuchen, ob eine KPT, die kognitiv-verhaltenstherapeutische Behandlungselemente umfasst, die ihre Effizienz bereits in Therapiestudien bestätigt haben, wirksamer in der Rückfallprophylaxe ist als eine reine Information durch ein Buch verbunden mit einer KurzPsychoedukation.

\section{Methodik}

\section{Patientenselektion}

Die Untersuchung wurde an der Verhaltenstherapieambulanz der Universitätsklinik für Psychiatrie und Psychotherapie, klinische Abteilung für Sozialpsychiatrie der Medizinischen Universität Wien in den Jahren 2004 bis 2008 durchgeführt. Ein Informationsbrief über die Studie wurde an alle niedergelassenen FachärztInnen für Psychiatrie und an alle psychiatrischen Abteilungen in Wien gesandt. Die Rekrutierung erfolgte nach Zuweisung durch niedergelassene FachärztInnen und/oder aus ambulanten und stationären psychiatrischen Einrichtungen (Sozialmedizinisches Zentrum-Ost, Kaiser-Franz-Josef-Spital, OttoWagner-Spital, Universitätsklinik für Psychiatrie im Allgemeinen Krankenhaus). Ein unabhängiger Interviewer stellte die grundsätzliche Eignung zur Teilnahme an der Studie fest (Prüfung von Ein- und Ausschlusskriterien) und führte die Baseline-Erhebung durch. Die PatientInnen wurden über die Studie im Detail informiert und gaben eine schriftliche Einverständniserklärung ab. Die Einschlusskriterien waren das Vorliegen einer Diagnose Bipolar-I- oder BipolarII-Störung nach der International Classification of Diseases 10 (ICD-10), eine stabile laufende medikamentöse Phasenprophylaxe und bestimmte Verlaufskriterien (in den vorangegangenen 3 Jahren mindestens 2 Krankheitsepisoden oder in 5 Jahren mindestens 3 Episoden). Alle PatientInnen mussten unabhängig von der klinischen Studie in einer regelmäßigen fachärztlichen medikamentösen Behandlung stehen und von dort aus z.B. notwendige Blutspiegelkontrollen der Phasenprophylaktika durchführen lassen.

Die PatientInnen sollten bei Studienbeginn außerhalb akuter manischer oder depressiver Episoden sein: Dafür wurden die Bech-Rafaelsen-Manie-Skala 
(BRMAS) [Bech et al., 1978], Cut-off-Wert: 9, und die Bech-Rafaelsen-Melancholie-Skala (BRMES) [Bech et al., 1980], Cut-off-Wert: 26, eingesetzt.

Ausschlusskriterien waren außerdem Suizidalität, das Vorliegen einer aktuellen psychotischen Episode oder die primäre Diagnose einer Suchterkrankung Insgesamt kontaktierten uns nach Zuweisung durch niedergelassene Fachärzte für Psychiatrie oder Spitalsärzte aus psychiatrischen Abteilungen 128 PatientInnen, mit denen die Eingangsuntersuchung durchgeführt wurde. Davon kamen 28 PatientInnen nicht für unsere Studie infrage: Bei 10 von diesen war die Diagnose bipolare Störung nicht erfüllt, 6 waren in einer akuten Krankheitsepisode (Überschreiten der Cut-off-Scores der Manie- bzw. Depressionsskalen), 12 PatientInnen schieden aus anderen Gründen aus (z.B. keine Phasenprophylaxe, gleichzeitige Einzelpsychotherapie, Zeitstruktur für die Gruppentherapie ungünstig, Entfernung zur Klinik zu groß). Die verbliebenen 100 Patienten wurden randomisiert den beiden Therapiegruppen zugeteilt: 52 in die KPT-Gruppe und 48 in die BT-Gruppe.

Die Studie wurde von der Ethikkommission der Medizinischen Universität Wien approbiert.

\section{Behandlungsprogramm und Ablauf der Studie}

Die PatientInnen wurden mit einem computerbasierten ZufallsstichprobenProgramm entweder der KPT-Gruppe oder der BT-Gruppe zugeteilt.

Das Behandlungsprogramm der KPT wurde nach dem Therapiemanual zur KPT bei bipolaren Erkrankungen von Schaub et al. [2004] durchgeführt. Die Inhalte dieses Programms betreffen einerseits Psychoedukation über Erkrankungen und Behandlung (Erklärungsmodell der bipolaren Erkrankung, Symptomatik und Verlauf, Informationen über Medikamente und ihre Nebenwirkungen, Symptomatik, Frühwarnsymptome und Bewältigungsmöglichkeiten bei Depression und Manie, gesunde Lebensführung usw.) und andererseits spezifische kognitiv-verhaltenstherapeutische Techniken (Aktivitätenaufbau, kognitive Strategien bei Depression und Manie, Vorbeugung von Rückfällen, Erhöhen der Belastbarkeit, Kommunikationsstrategien usw.).

Die KPT-Gruppensitzungen fanden in einem Zeitraum von 14 Wochen mit insgesamt 14 wöchentlichen Sitzungen zu je 90 min statt. Boostersitzungen wurden jeweils 6 und 9 Monate nach Studienbeginn durchgeführt, eine Nachunter suchung erfolgte 12 Monate nach Studienbeginn. Die Anzahl der GruppenteilnehmerInnen betrug meist 10 Personen pro Gruppe. Zusätzlich wurde für die Angehörigen der KPT-Gruppe auch eine Angehörigengruppe in 4 Sitzungen über insgesamt $8 \mathrm{~h}$ durchgeführt.

Gemäß des Behandlungsprogramms der BT-Gruppe bekamen die PatientInnen ein Informationsbuch über bipolare Störungen überreicht [Buch: «Sturzfliegen - Leben in Depressionen und Manien» von Vasak und Katschnig, 2001] mit der Aufforderung, das Buch zu lesen und sich daraus ergebende Fragen für die BT-Gruppensitzungen zu überlegen.

Das Buch wurde auch deshalb ausgewählt, weil hier 3 Ebenen der bipolaren Störung dargestellt werden: Die Innenseite am Beispiel der Romanfigur Simon, der Alltag der Betroffenen durch zahlreiche Interviews mit realen PatientInnen und die fachliche Ebene aus der Sicht eines Experten. Es fanden 3 Gruppensitzungen zu je 90 min mit Vortrag und Diskussionsrunden unter fachärztlicher Leitung mit Informationen über Krankheit und Behandlung statt (Zeitpunkt 0, nach 1 Monat, nach 3 Monaten), mit Auffrischungssitzungen 6 und 9 Monate nach Studienbeginn.

$\mathrm{Zu}$ den einzelnen Treffen wurden auch Angehörige und/oder eine nahestehende Bezugsperson eingeladen.

Drei unabhängige Beurteiler (FachärztInnen für Psychiatrie) führten $12 \mathrm{Mo}$ nate nach Beginn der Intervention eine Nachuntersuchung mit einem strukturierten Interview (Mini International Neuropsychiatric Interview (MINI)) durch und erfassten, ob es in den vergangenen 12 Monaten zu Rückfällen gekommen war (Art und Häufigkeit von Rückfällen und Anzahl und Dauer von Spitalsaufenthalten in der Psychiatrie), beurteilten den Eindruck nach der Clinical Global Impression (CGI)-Skala und die Resultate des Medication Compliance Questionnaire (MCQ) und dokumentierten die Ergebnisse der Krankheitskonzept-Skala (KK-Skala) und der Sheehan-Skala.

Die Gruppentherapeutinnen $(\mathrm{n}=4)$ der KPT-Gruppen waren alle Psychologinnen mit abgeschlossener Verhaltenstherapieausbildung, die Gruppensitzung zur Medikation wurde gemeinsam mit einem Facharzt abgehalten. Die BT-
Gruppen wurden von einem Facharzt für Psychiatrie und psychotherapeutische Medizin geleitet, die Nachuntersuchungen wurden von Fachärzten für Psychiatrie und psychotherapeutische Medizin durchgeführt, die in Bezug auf die Gruppenzuteilungen blind waren.

\section{Untersuchungsinstrumente}

Das MINI [Sheehan et al., 1998], ein kurzes strukturiertes psychiatrisches Interview zur Erstellung von Diagnostic and Statistic Manual of Mental Disorders (DSM)-IV- und ICD-10-Diagnosen, wurde am Beginn und beim 12-Monats-Follow-up zur Erstellung der Diagnose und Beurteilung von Rückfällen durchgeführt. Als Rückfall wurde eine Episode definiert, die die ICD-10- und DSM-IV-Kriterien für eine manische, depressive oder gemischte Episode erfüllten. Des Weiteren wurden im Verlauf der Studie auch ein Strukturiertes Klinisches Interview für DSM-IV (SKID)-II-Fragebogen (Selbstrating zum Screening) und ein SKID-II-Interview [Fydrich et al., 1997] zur Diagnose von Persönlichkeitsstörungen durchgeführt. Neben einem Dokumentationsbogen zu soziodemografischen Daten (Name, Alter, Geschlecht, Zivilstand, Schulbildung, Beruf, Lebensunterhalt) wurde auch ein Dokumentationsbogen für Krankheitsdaten ausgefüllt: Diagnose auf Achse I und II einschließlich Komorbidität, Alter bei Beginn der Erkrankung, Krankheitsdauer, Art und Gesamtdauer der Phasenprophylaxe, Typisierung des Krankheitsverlaufes (episodisch mit oder ohne freie Intervalle, Rapid Cycling), bisheriger Erfolg der Phasenprophylaxe, Compliance in den letzten 2 Jahren, derzeitige und frühere Phasenprophylaxe und Gründe für etwaiges Absetzen, Life-Chart über Anzahl und Art von Krankheitsepisoden und Anzahl und Dauer von stationären Aufenthalten in einem Zeitraum von 12 Monaten vor und nach Beginn der Gruppenintervention.

Die Compliance für die Medikation und die Nebenwirkungen der Medikation wurden mit dem MCQ [Lam et al., 2000] bei Beginn sowie nach 3 und nach 12 Monaten gemessen (Befragung durch die UntersucherInnen, wie regelmäßig die Phasenprophylaxe in den letzten 4 Wochen eingenommen wurde: Als gute Compliance wurde angesehen, wenn die PatientInnen niemals oder maximal 1bis 2-mal die Medikation nicht eingenommen hatten, als schlechte Compliance, wenn sie öfters als 2-mal nicht eingenommen oder überhaupt abgesetzt hatten).

Die CGI-Skala [NIMH, 1976] ist eine Fremdrating-Skala und dient der Bewertung des Erfolgs psychopharmakologischer Behandlungen durch die Globalbeurteilung eines Klinikers. Beurteilt werden die Schwere der Erkrankung, die Zustandsänderung über die Zeit sowie die therapeutische Wirksamkeit und die unerwünschten Wirkungen einer Behandlung. Die CGI-Skala wurde hier nur zur Gesamtbeurteilung der Zustandsänderung nach 12 Monaten (von $1=\mathrm{Zu}$ stand ist sehr viel besser bis $7=$ Zustand ist sehr viel schlechter) verwendet.

Die KK-Skala von Linden et al. [1988] ist eine Selbstbeurteilungsskala und gibt Auskunft über die subjektive Beurteilung von Krankheitskonzepten von PatientInnen (ursprünglich für PatientInnen mit schizophrenen Störungen) und wurde hier unverändert für die PatientInnen mit bipolaren affektiven Störungen verwendet. Die Skala erlaubt die Messung von 7 Krankheitskonzept-Dimensionen: Medikamentenvertrauen, Arztvertrauen, Negativerwartungen, Schuld, Zufallskontrolle, Anfälligkeit, idiosynkratische Annahmen.

Bei der Sheehan-Skala zu Behinderung/Beeinträchtigung (Sheehan Disability Scale (SDS)) [Sheehan and Harnett-Sheehan Raj, 1996] handelt es sich um eine Selbstbeurteilungsskala. Mittels der SDS werden auf einer 10-PunkteLikert-Skala die Funktionsbeeinträchtigungen im letzten Monat (von $0=$ überhaupt nicht bis $10=$ extrem) für 3 Bereiche erfasst: Arbeit und Beruf, Sozialkontakte und Freizeitverhalten sowie Familienleben und häusliche Verpflichtungen. Des Weiteren wurden die BRMAS [Bech et al., 2005] und die BRMES [Bech et al., 1980, 1998] verwendet.

\section{Datenanalyse}

Die statistischen Analysen erfolgten mit dem Programmpaket SPSS (Version 17.0). Das $\alpha$-Niveau wurde auf $5 \%$ festgesetzt. Alle Hauptanalysen wurden als Intent-to-Treat(ITT)-Analysen durchgeführt. Folgende Analyseverfahren kamen zur Anwendung: deskriptiv statistische Verfahren: Mittelwert $(\mu)$, Standardabweichung ( $\sigma$ ), Spannbreite (Range, []), Median, Konfidenzintervall (KI) sowie Prozentangaben. Gruppenunterschiede wurden mittels multivariater Varianzanalysen (inklusive Box-M-Test), t-Tests (für abhängige bzw. unabhängige Stichproben und homogene bzw. heterogene Varianzen) und Mann-Whitney- 
Tab. 1. Stichprobenbeschreibung vor Beginn der Behandlung

\begin{tabular}{|c|c|c|c|c|}
\hline & $\begin{array}{l}\text { Total sample } \\
\mathrm{n}=100\end{array}$ & $\begin{array}{l}\text { KPT } \\
\mathrm{n}=52\end{array}$ & $\begin{array}{l}\text { BT } \\
\mathrm{n}=48\end{array}$ & Statistik \\
\hline Geschlecht weiblich & $59(59 \%)$ & $31(59,6 \%)$ & $28(58,3 \%)$ & $\chi^{2}: p=0,896$ n.s. \\
\hline Alter $\overline{\mathrm{x}}$, Jahre & $40 \pm 11,1$ & $38,5 \pm 10,6$ & $41,7 \pm 11,5$ & t-Test: $\mathrm{p}=0,156$ n.s. \\
\hline Diagnose BP I/BP II & $76 / 24$ & $40 / 12$ & $36 / 12$ & $\chi^{2}: p=0,822$ n.s. \\
\hline Komorbidität Achse 1 & 33 & 19 & 14 & $\chi^{2}: p=0,433$ n.s. \\
\hline Komorbidität Achse 2 & 41 & 25 & 16 & $\chi^{2}: p=0,134$ n.s. \\
\hline Krankheitsdauer $\overline{\mathrm{x}}$, Monate & $182,7 \pm 131,1$ & $164,6 \pm 110,3$ & $202,3 \pm 149,2$ & $\mathrm{t}$-Test: $\mathrm{p}=0,151 \mathrm{n} . \mathrm{s}$ \\
\hline Rapid Cycling bisher im Verlauf & 16 & 7 & 9 & $\chi^{2}: p=0,471$ n.s. \\
\hline $\begin{array}{l}\text { Anzahl der depressiven Episoden } \\
\text { (12 Monate vor Therapie) }\end{array}$ & 87 & 47 & 40 & U-Test: $\mathrm{p}=0,954$ n.s. \\
\hline $\begin{array}{l}\text { Anzahl der hypomanischen/manischen } \\
\text { Episoden ( } 12 \text { Monate vor Therapie) }\end{array}$ & 63 & 36 & 23 & U-Test: $\mathrm{p}=0,424$ n.s. \\
\hline Bildungsniveau Matura (Abitur) oder höher & $64(64 \%)$ & $37(71,2 \%)$ & $27(56,3 \%)$ & $\chi^{2}: p=0,121$ n.s. \\
\hline Einnahme von Valproinsäure & $43(43 \%)$ & $27(51,9 \%)$ & $16(33,3 \%)$ & $\chi^{2}: p=0,061$ n.s. \\
\hline Einnahme von Lamotrigin & $34(34 \%)$ & $17(32,7 \%)$ & $17(35,4 \%)$ & $\chi^{2}: p=0,774$ n.s. \\
\hline Einnahme von Lithium & $23(23 \%)$ & $12(23,1 \%)$ & $11(22,9 \%)$ & $\chi^{2}: p=0,985$ n.s. \\
\hline Einnahme von Carbamazepin & $11(11 \%)$ & $6(11,5 \%)$ & $5(10,4 \%)$ & $\chi^{2}: p=0,858$ n.s. \\
\hline Einnahme von atypischen Neuroleptika & $46(46 \%)$ & $21(40,4 \%)$ & $25(52,1 \%)$ & $\chi^{2}: p=0,241$ n.s. \\
\hline Schlechte Compliance in den letzten 2 Jahren & $49(49 \%)$ & $27(51,9 \%)$ & $22(45,8 \%)$ & $\chi^{2}: p=0,625$ n.s. \\
\hline BRMAS $\bar{x}$ & $1,6 \pm 2,7$ & $1,8 \pm 2,8$ & $1,4 \pm 2,6$ & t-Test: $\mathrm{p}=0,384$ n.s. \\
\hline BRMES $\bar{x}$ & $7,2 \pm 5,1$ & $7,4 \pm 5,4$ & $6,9 \pm 4,8$ & $\mathrm{t}$-Test: $\mathrm{p}=0,622$ n.s. \\
\hline
\end{tabular}

U-Tests geprüft. Zur Abklärung von Verteilungsunterschieden wurden Kreuztabellen (inklusive $\chi^{2}$-Test) erstellt. Die statistische Beratung und Auswertung erfolgte durch die Statistikambulanz KG (Dr. Andrea Schrott).

\section{Ergebnisse}

Tabelle 1 zeigt die Baseline-Daten der in die Studie eingegangenen 100 PatientInnen. Die Komorbidität auf Achse II nach DSMIV betraf vor allem Borderline-Persönlichkeitsstörungen $(\mathrm{n}=14)$, anankastische und vermeidende (jeweils $n=6$ ) und paranoide und histrionische Persönlichkeitsstörungen (jeweils $n=4$ ). 16\% Rapid Cycling zeigt, dass es hier eine beachtenswert große Gruppe mit besonders schwierigem Verlauf gibt. Die Zahl von insgesamt 150 Krankheitsepisoden im letzten Jahr vor Beginn der Studie bei den 100 PatientInnen lässt hohe Rückfallraten im weiteren Jahr befürchten. Neben den PatientInnen nahmen auch insgesamt 95 Angehörige an der Studie teil, die sich 87 verschiedenen PatientInnen zuordnen ließen. 56\% waren Angehörige der PatientInnen der KPT-Gruppe und 44\% Angehörige der PatientInnen der BT-Gruppe. Insgesamt waren $48 \%$ der Angehörigen aus der Ursprungsfamilie, 37\% PartnerInnen und 15\% Freunde bzw. andere.

Im vorausgegangenen Krankheitsverlauf hatten 45 PatientInnen jemals eine Phasenprophylaxe mit Lithium eingenommen (davon 66,7\% irgendwann zumindest 1-mal abgesetzt), 63 Valproinsäure (davon 46,0\% irgendwann abgesetzt), 36 Lamictal (36,1\% abgesetzt), 25 Carbamazepin (64,0\% abgesetzt) und 57 atypische Antipsychotika (33,3\% abgesetzt). Gründe für das Absetzen waren Nebenwirkungen, eine negative Einstellung gegenüber der Medikation, beeinträchtigtes Wohlbefinden oder auch mangelnde Wirksamkeit.
Tab. 2. Therapeutische Wirksamkeit nach 12 Monaten

\begin{tabular}{lll}
\hline CGI & $\begin{array}{l}\text { KPT } \\
\mathrm{n}=49\end{array}$ & $\begin{array}{l}\text { BT } \\
\mathrm{n}=47\end{array}$ \\
\hline Sehr viel/viel besser & 37 & 26 \\
Wenig besser/unverändert & 12 & 21 \\
\hline
\end{tabular}

CGI = Clinical Global Impression; KPT = kognitiv-psychoedukative Therapie; $\mathrm{BT}=$ Bibliotherapie.

Pearson $\chi^{2}=4,3 ; \mathrm{df}=1 ; \mathrm{p}=0,037$.

\section{Outcome 12 Monate nach Beginn der Gruppentherapie}

Ein 12-Monats-Follow-up konnte in der KPT-Gruppe bei 45 von 52 PatientInnen durchgeführt werden, in der BT-Gruppe bei 40 von 48 PatientInnen.

Zur Regelmäßigkeit des Gruppenbesuchs: In einem ambulanten Setting ist es unrealistisch, dass alle Teilnehmer eine 100\%ige Anwesenheit erreichen.

In der kognitiv-psychoedukativen Gruppe nahmen 47 der 52 PatientInnen (90\%) an mindestens zwei Dritteln der Gruppentherapiesitzungen teil, in der BT-Gruppe 37 der 48 PatientInnen (77\%). Tabelle 2 zeigt die globale Einschätzung der Zustandsänderung (nach CGI) nach 12 Monaten: Die PatientInnen der KPTGruppe schneiden hier signifikant besser ab als jene der BT-Gruppe. Wenn man die Anzahl der Krankheitsepisoden und die Anzahl und Dauer von Krankenhausaufnahmen betrachtet (Tab. 3) 12 Monate vor im Vergleich zu 12 Monate nach Studienbeginn, zeigt sich eine signifikante Reduktion der Gesamtzahl von Krankenhausepisoden, d.h. in der Zahl und Dauer der Krankenhausaufenthalte, in beiden Gruppen. 
Tab. 3. Anzahl der Krankheitsepisoden/ Krankenhausaufenthalte im Beobachtungszeitraum 12 Monate vor (T0) bzw. nach Studienbeginn (T12)

Tab. 4. Veränderungen im Krankheitskonzept (Linden-Skala) T0-T12
Tab. 5. ComplianceMCQ für Phasenprophylaxe zu Studienbeginn (T0) bzw. nach 12 Monaten (T12)

\begin{tabular}{|c|c|c|c|c|c|c|}
\hline & \multicolumn{3}{|l|}{ KPT } & \multicolumn{3}{|l|}{ BT } \\
\hline & $\begin{array}{l}\text { T0 } \\
(\mathrm{n}=50-52)\end{array}$ & $\begin{array}{l}\text { T12 } \\
(\mathrm{n}=45-49)\end{array}$ & $\mathrm{p}^{*}$ (Zeit) & $\begin{array}{l}\text { T0 } \\
(\mathrm{n}=45-48)\end{array}$ & $\begin{array}{l}\text { T12 } \\
(\mathrm{n}=40-47)\end{array}$ & $\mathrm{p}^{*}$ (Zeit) \\
\hline & $\mu \pm \sigma$ & $\mu \pm \sigma$ & & $\mu \pm \sigma$ & $\mu \pm \sigma$ & \\
\hline Krankheitsepisoden gesamt, $\mathrm{n}$ & $2,48 \pm 2,10$ & $1,51 \pm 2,18$ & $0,000^{*}$ & $2,65 \pm 2,39$ & $2,23 \pm 2,61$ & $0,034^{*}$ \\
\hline Manische Episoden, $\mathrm{n}$ & $0,80 \pm 0,70$ & $0,36 \pm 0,61$ & $0,000^{*}$ & $0,73 \pm 0,72$ & $0,20 \pm 0,41$ & $0,001^{*}$ \\
\hline Depressive Episoden, $\mathrm{n}$ & $1,00 \pm 0,88$ & $0,58 \pm 0,87$ & $0,003^{*}$ & $0,84 \pm 0,67$ & $1,00 \pm 0,88$ & n.s. \\
\hline Krankenhausaufnahmen, $\mathrm{n}$ & $1,13 \pm 1,57$ & $0,35 \pm 0,75$ & $0,000^{*}$ & $0,77 \pm 0,87$ & $0,30 \pm 0,55$ & $0,002^{*}$ \\
\hline $\begin{array}{l}\text { Dauer Krankenhausaufnahmen, } \\
\text { Tage (gesamt) }\end{array}$ & $34,02 \pm 40,77$ & $12,10 \pm 28,89$ & $0,000^{*}$ & $27,19 \pm 33,38$ & $12,45 \pm 31,27$ & $0,013^{*}$ \\
\hline
\end{tabular}

\begin{tabular}{|c|c|c|c|c|c|c|}
\hline \multirow[t]{2}{*}{ KK-Skala, Mittelwerte } & \multicolumn{2}{|l|}{ KPT } & \multicolumn{2}{|l|}{ BT } & \multirow{2}{*}{$\frac{\text { Zeiteffekt }}{\mathrm{p}}$} & \multirow{2}{*}{$\begin{array}{l}\text { Gruppeneffekt } \\
\mathrm{p}\end{array}$} \\
\hline & T0 & T12 & T0 & T12 & & \\
\hline $\begin{array}{l}\text { Skala 1: } \\
\text { Medikamentenvertrauen }\end{array}$ & 3,13 & 3,30 & 2,79 & 3,04 & $0,001^{*}$ & n.s. \\
\hline $\begin{array}{l}\text { Skala 2: } \\
\text { Arztvertrauen }\end{array}$ & 2,94 & 3,06 & 2,70 & 2,83 & $0,039^{*}$ & n.s. \\
\hline $\begin{array}{l}\text { Skala 3: } \\
\text { Negativerwartungen }\end{array}$ & 1,15 & 1,10 & 1,59 & 1,54 & n.s. & n.s. \\
\hline $\begin{array}{l}\text { Skala 4: } \\
\text { Schuld }\end{array}$ & 1,26 & 1,11 & 1,64 & 1,69 & n.s. & n.s. \\
\hline $\begin{array}{l}\text { Skala 5: } \\
\text { Zufallskontrolle }\end{array}$ & 1,40 & 1,57 & 1,52 & 1,50 & n.s. & n.s. \\
\hline $\begin{array}{l}\text { Skala 6: } \\
\text { Anfälligkeit }\end{array}$ & 2,07 & 2,25 & 2,19 & 2,15 & n.s. & n.s. \\
\hline $\begin{array}{l}\text { Skala 7: } \\
\text { Idiosynkratische Annahmen }\end{array}$ & 1,40 & 1,03 & 1,71 & 1,44 & $0,003^{*}$ & n.s. \\
\hline
\end{tabular}

KK-Skala = Krankheitskonzept-Skala; KPT = kognitiv-psychoedukative Therapie; BT = Bibliotherapie; n.s. = nicht signifikant .

\begin{tabular}{|c|c|c|c|c|c|c|}
\hline & \multicolumn{2}{|l|}{ KPT } & \multicolumn{2}{|l|}{ BT } & \multicolumn{2}{|c|}{ Häufigkeitsverteilung } \\
\hline & T0 & $\mathrm{T} 12$ & T0 & $\mathrm{T} 12$ & & \\
\hline & & & & & Kreuztabellen & $\mathrm{p}\left(\chi^{2}\right)$ \\
\hline Phasenprophylaxe & $\mathrm{n}=50$ & $\mathrm{n}=37$ & $\mathrm{n}=46$ & $\mathrm{n}=41$ & & \\
\hline Gute Compliance & $92,0 \%$ & $86,5 \%$ & $89,1 \%$ & $78,0 \%$ & KPT: Zeit & n.s. \\
\hline Schlechte Compliance & $8,0 \%$ & $13,5 \%$ & $10,9 \%$ & $22,0 \%$ & BT: Zeit & $0,030^{*}$ \\
\hline
\end{tabular}

Wenn man die Anzahl manischer und depressiver Episoden getrennt untersucht, dann zeigt sich in der KPT-Gruppe eine signifikante Reduktion von beiden Episoden, in der BT-Gruppe aber nur eine Reduktion der manischen Episoden, nicht der depressiven Episoden.

Tabelle 4 beschreibt die Veränderung von Krankheitskonzepten nach einem Zeitraum von 12 Monaten. Die Prüfung der Mittelwertsunterschiede mittels $2 \times 2$ Varianzanalyse (Zeit $\times$ Therapieform/Gruppe) war aufgrund der Verletzung der Modellvoraussetzungen (Homogenität der Kovarianzmatrix, geprüft mittels BoxM-Test) nicht zulässig. Es zeigt sich insgesamt eine Erhöhung der Dimension Medikamentenvertrauen (Skala 1: Vertrauen in die
Hilfestellung durch Medikamente und Vertrauen in die Wirksamkeit) und der Dimension Arztvertrauen (Skala 2: Vertrauen in die wirksame Hilfestellung durch den Arzt) und eine Abnahme in der Dimension idiosynkratische Annahmen (Skala 7: dysfunktionale Annahmen über die Krankheit und die Bedeutung der medikamentösen Therapie), d.h. innerhalb jeder Gruppe ist ein Zeiteffekt feststellbar, es gibt aber keine Gruppenunterschiede.

In Tabelle 1 ist die Compliance für die Phasenprophylaxe für die letzten 2 Jahre vor Studienbeginn dargestellt, wobei eine Einnahme über mehr als $90 \%$ der Zeit als gute Compliance gewertet wurde. Nur 40\% erreichten hier eine regelmäßige Einnahme, 49\% eine unregelmäßige Einnahme und bei 11\% war es unbekannt bzw. 
Tab. 6. Sheehan-Skala - Behinderungs-Beeinträchtigungsskala (BBS): zum Studienbeginn (T0) bzw. nach 12 Monaten (T12)

\begin{tabular}{|c|c|c|c|c|}
\hline \multirow{2}{*}{$\begin{array}{l}\text { BBS, Skalen bzw. } \\
\text { Prozentränge }\end{array}$} & \multicolumn{2}{|l|}{$\mathrm{KPT}, \mu \pm \sigma$} & \multicolumn{2}{|l|}{ BT, $\mu \pm \sigma$} \\
\hline & T0 $(\mathrm{n}=49)$ & $\mathrm{T} 12(\mathrm{n}=33)$ & $\mathrm{T} 0(\mathrm{n}=41)$ & $\mathrm{T} 12(\mathrm{n}=41)$ \\
\hline Skala 1: Arbeit & $5,8 \pm 3,0$ & $3,5 \pm 3,2$ & $5,2 \pm 3,3$ & $4,1 \pm 3,0$ \\
\hline Skala 2: Freizeit & $5,4 \pm 3,0$ & $3,6 \pm 2,9$ & $5,2 \pm 3,2$ & $3,7 \pm 2,9$ \\
\hline \multirow[t]{2}{*}{ Skala 3: Familie } & $4,8 \pm 3,2$ & $2,9 \pm 2,8$ & $4,5 \pm 3,2$ & $3,8 \pm 3,2$ \\
\hline & $\%$ & $\%$ & $\%$ & $\%$ \\
\hline \multicolumn{5}{|l|}{ Über Cut-off in } \\
\hline 0 Skalen & 23,4 & 51,5 & 23,1 & 42,3 \\
\hline 1 Skala & 12,8 & 6,1 & 20,5 & 11,5 \\
\hline 2 Skalen & 21,3 & 18,2 & 12,8 & 23,1 \\
\hline 3 Skalen & 42,6 & 24,2 & 43,6 & 23,1 \\
\hline
\end{tabular}

Tab. 7. SheehanSkala - BehinderungsBeeinträchtigungsskala (BBS): multivariate Varianzanalyse

\begin{tabular}{ll}
\hline Effekte & $\mathrm{p}($ Wilk's $\lambda)$ \\
\hline Gruppe & n.s. \\
Skala & $0,017^{\star}$ \\
Zeit & $0,001^{\star}$ \\
Zeit $\times$ Gruppe & $0,086^{+}$ \\
Skala $\times$Gruppe & n.s. \\
Skala $\times$Zeit & n.s. \\
Skala $\times$Zeit $\times$Gruppe & n.s. \\
\hline n.s. $=$ Nicht signifikant. & \\
\hline
\end{tabular}

nicht zutreffend (z.B. noch keine 2 Jahre Phasenprophylaxe). Tabelle 5 zeigt die Compliance für die Phasenprophylaxe zu Studienbeginn und zum Nachuntersuchungszeitpunkt nach dem MCQ. Zum Zeitpunkt des Studienbeginns war die Compliance bereits in beiden Gruppen relativ hoch und nahm nur in der BT-Gruppe nach 1 Jahr wieder signifikant ab.

Tabellen 6 und 7 zeigen die Ergebnisse für die BehinderungsBeeinträchtigungsskala (Sheehan-Skala): Hier ließ sich eine Veränderung über die Zeit für alle Skalen (wenn auch in unterschiedlichem Ausmaß) zeigen, diese ist jedoch unabhängig von der Gruppe (signifikanter Zeit- und Skaleneffekt, kein signifikanter Gruppeneffekt). Während in der KPT und der BT zum Zeitpunkt T0 42,6\% bzw. 43,6\% der PatientInnen über dem Cut-off in allen 3 Skalen liegen, sind es zu T12 nur noch 24,2\% bzw. 23,1\%. Diese Reduktion von Behinderung und Beeinträchtigung ist ein wichtiger Erfolgsfaktor im psychosozialen Bereich.

\section{Diskussion}

Unsere Untersuchung unterstreicht die Wirksamkeit der Anwendung des deutschsprachigen KPT-Manuals in der Behandlung bei bipolaren Patienten. In unserer BT-Gruppe (die man als Kombination von Information und Kurz-Psychoedukation in der Gruppe sehen kann) sollte das Lesen eines ausführlichen Buches über bipolare Störungen die geringere Therapiedauer von nur 3 Gruppensitzungen ausgleichen, wobei die Ergebnisse allerdings keinen Einfluss auf die Reduktion depressiver Rückfälle zeigten. Hier scheinen die kognitiv-verhaltenstherapeutischen Elemente in der KPT-Gruppe doch wichtig in der Verhinderung depressiver Rückfälle zu sein. Ähnlich berichten Bond et al. [2015] in einem neueren Überblick über 16 randomisiert-kontrollierte Studien, dass Psychoedukation allein offenbar gute Effekte bezüglich einer Verringerung der Rückfallhäufigkeit für manische, aber nicht für depressive Phasen zeigt. Die Verbesserungen im Krankheitskonzept nach Linden für beide Gruppen können einerseits durch die kognitiven Elemente in der KPT-Gruppe erklärt werden, andererseits scheint in der BT-Gruppe das Lesen von Informationen über die Erkrankung und Behandlung einschließlich der eindrücklichen Selbstschilderungen von PatientInnen mit nachfolgender Gruppendiskussion ähnliche Einstellungsänderungen bewirken zu können. Informationsvermittlung findet neben Büchern heute ja sehr viel mehr über das Internet statt, wobei vor allem im angloamerikanischen Sprachraum auch Online-Self-Management-Programme angeboten werden, wie Leitan et al. [2015] in ihrem Überblick darstellen. In einem Vergleich zweier australischer Internet-Selbsthilfe-Programme in einer Studie von Lauder et al. [2015] zeigten sich in beiden Gruppen eine Verbesserung der Adhärenz für die Medikation und Verbesserungen in der Symptomatik und der Lebensqualität nach 12 Monaten.

Der Einfluss der Behandlung auf die Adhärenz für die Medikation war auch für uns ein wichtiges Untersuchungsanliegen. Die große Differenz zwischen der eher schlechten Compliance in den letzten 2 Jahren vor Studienbeginn (Tab. 1) und der guten Compliance in den letzten 4 Wochen vor Studienbeginn (MCQ) könnte einerseits dadurch erklärt werden, dass viele PatientInnen im Jahr vor der Gruppentherapie wegen schlechter Compliance und Rückfällen in stationärer Behandlung und dadurch bereits wieder motivierter für die Phasenprophylaxe bei Studienbeginn waren, und andererseits vielleicht dadurch, dass die zuweisenden ÄrztInnen ein besonderes Augenmerk auf die Überwachung der von uns geforderten regelmäßigen Phasenprophylaxe legten. Das heißt, es ging eher darum, die gute Compliance zu Studienbeginn über die nächsten 12 Monate zu halten, als eine schlechte Compliance zu verbessern, und das gelang vor allem in der KPT-Gruppe.

Als mögliche Einschränkung der Studienergebnisse kann angeführt werden, dass unsere PatientInnen eine Selektion von Hochri- 
sikopatienten mit langer mittlerer Krankheitsdauer und hoher Rückfallneigung darstellten. Als wesentliches Outcome-Kriterium für unsere Studie verwendeten wir die Veränderung der Episodenzahl in gleichen Zeiträumen vor und nach Beginn der Therapie, wobei bei bewusster Selektion von Patienten mit großer Episodenzahl in den letzten 3-5 Jahren vor Beginn der Intervention sicherlich der «Regression-to-the-Mean-Effekt» bedacht werden muss [Linden, 2013]. Dieser Begriff bezeichnet das Phänomen, dass nach einem extrem ausgefallenen Messwert die nachfolgende Messung wieder näher am Durchschnitt liegt, falls der Zufall einen Einfluss auf die Messgröße hat. Andererseits ist aus Verlaufsstudien bekannt, dass bei bipolaren Störungen die Episodenhäufigkeit im Verlauf eher zu- als abnimmt [Brieger und Marneros, 2006].
Das Manual von Schaub et al. [2004] ist aber auf jeden Fall eine wertvolle und wichtige Hilfestellung sowohl in der ambulanten Praxis als auch im stationären Rahmen. Wenn eine solche Therapie aus bestimmten Gründen nicht möglich bzw. nicht erreichbar ist, werden in Zukunft Informationsangebote wie BT oder internetbasierte Informationen und Programme vermehrt Bedeutung erlangen, wobei deren Wirksamkeit bzw. deren wirksame Bestandteile noch genauer erforscht werden müssen.

\section{Disclosure Statement}

Die Autoren erklären, dass keine Interessenkonflikte vorliegen.

\section{Literatur}

Bech P, Rafaelsen OJ, et al: The mania rating scale. Neuropharmacology 1978;17:430-431 (deutsche Übersetzung: «CIPS-Skalen». Göttingen, Beltz-Test, 1996 und 2005).

Bech P, Rafaelsen OJ: The use of rating scales exemplified by a comparison of the Hamilton and the Bech-Rafaelsen Melancholia Scale. Acta Psychiatr Scand 1980; 62(suppl 285):128-131 (deutsche Version: Stieglitz RD, et al: Bech-Rafaelsen-Melancholie-Skala (BRMS). Göttingen, Hogrefe, 1998).

Bernhard B: Wirksamkeit einer kognitiv-psychoedukativen Gruppenintervention bei bipolaren Patienten. Dissertation LMU München, Medizinische Fakultät, 2009.

Bond K, Anderson IM: Psychoeducation for relapse prevention in bipolar disorder: a systematic review of efficacy in randomized controlled trials. Bipolar Disord 2015;17:349-362.

Brieger P, Marneros A: Prognose; in Bauer M (ed): Weißbuch Bipolare Störungen in Deutschland. Hamburg, Deutsche Gesellschaft für Bipolare Störungen, 2006, pp 283-324.

DGPPN (Deutsche Gesellschaft für Psychiatrie und Psychotherapie, Psychosomatik und Nervenheilkunde): S3-Leitlinie zur Diagnostik und Therapie bipolarer Störungen, Langversion 1.8; Mai 2012, letzte Anpassung Januar 2014. www.leitlinie-bipolar.de (zuletzt heruntergeladen am 1. November 2014).

Erfurth A, Dobmeier M, Zechendorff M: Kurzpsychoedukation bei bipolaren Erkrankungen. Stuttgart, Thieme, 2005.

Fydrich T, Renneberg B, Schmitz B, Wittchen HU: SKID-II Strukturiertes Klinisches Interview für DSM-IV Achse II. Göttingen, Hogrefe, 1997.

Grahlmann K, Linden M: Bibliotherapie. Verhaltenstherapie 2005; 15:88-93.

Grunze H, Vieta E, Goodwin GM, et al: The World Federation of Societies of Biological Psychiatry (WFSBP) Guidelines for the Biological Treatment of Bipolar Disorders: Update on the long-term treatment of bipolar disorder. World J Biol Psychiatry 2013;14:154-219.
Hautzinger M, Meyer TD: Psychotherapie bei bipolaren affektiven Störungen. Ein systematischer Überblick kontrollierter Interventionsstudien. Nervenarzt 2007; 78:1248-1260

Herm K: Verhaltenstherapie integrierte Bibliotherapie: eine experimentelle Studie in der psychosomatischen Rehabilitation. Dissertation, Freie Universität Berlin, 2010.

Jelley R, Elmer OM: HOPE - Handlungsorientierte Psychoedukation bei Bipolaren Störungen. Tübingen, DGVT, 2005.

Lauder S, Chester A, Castle D, et al: A randomized head to head trial of MoodSwings.net.au: an Internet based self-help program for bipolar disorder. J Affect Disord 2015;171:13-21.

Leitan ND, Michalak EE, Berk L, et al: Optimizing delivery of recovery-oriented online self-management strategies for bipolar disorder: a review. Bipolar Disord 2015;17:115-127.

Linden M, Nather J, Wilms HU: Zur Definition, Bedeutung und Messung der Krankheitskonzepte von Patienten. Die Krankheitskonzeptskala (KK-Skala). Fortschr Neurol Psychiatr 1988;56:35-43.

Linden A: Assessing regression to the mean effects in health care initiatives. BMC Med Res Methodol 2013; 13:119.

Lynch D, Laws KR, McKenna PJ: Cognitive behavioural therapy for major psychiatric disorder: does it really work? A meta-analytical review of well-controlled trials. Psychol Med 2010;40:9-24.

Meyer TD, Hautzinger M: Manisch-depressive Störungen: Kognitiv-verhaltenstherapeutisches Behandlungsmanual. Weinheim, Beltz PVU, 2004.

Meyer TD, Hautzinger M: Cognitive behaviour therapy and supportive therapy for bipolar disorders: relapse rates for treatment period and 2-year follow-up. Psychol Med 2012;42:1429-1439.

NIMH (National Institute of Mental Health): Clinical Global Impressions; in Guy W (ed): ECDEU Assessment Manual for Psychopharmacology, ed 2. Rockville, NIMH, 1976, pp 218-222.
Niedermayer G: Bibliotherapie - eine Bestandsaufnahme in Zeiten des Internet. Diplomarbeit, FH Eisenstadt, 2006.

Reinares M, Sanchez-Moreno J, Fountoulakis KN: Psychosocial interventions in bipolar disorder: what, for whom and when: J Affect Disord 2014;156:46-55.

Schaub A, Bernhard B, Gauck L: Kognitiv-psychoedukative Therapie bei bipolaren Erkrankungen. Ein Therapiemanual. Göttingen, Hogrefe, 2004.

Schaub A, Neubauer N: Psychotherapie bei bipolaren Störungen - Therapiekonzepte, ihre Inhalte und Wirksamkeit. Fortschr Neurol Psychiatr 2013;81(suppl1): S22-S29.

Schaub A, Neubauer N, Bernhard B, et al: Kognitiv-psychoedukative Gruppenintervention bei bipolar erkrankten Patienten. Fortschr Neurol Psychiatr 2013; 81(suppl 1):S30-S34.

Schöttle D, Huber CG, Bock T, Meyer TD: Psychotherapy for bipolar disorder: a review of the most recent studies. Curr Opin Psychiatry 2011;24:549-555.

Sheehan DV, Harnett-Sheehan Raj BA: The measurement of disability. Int Clin Psychopharmacol 1996; (suppl 3): 89-95.

Sheehan DV, Lecrubier Y, Sheehan KH, et al: The MiniInternational Neuropsychiatric Interview (M.I.N.I.): the development and validation of a structured diagnostic psychiatric interview for DSM-IV and ICD-10. J Clin Psychiatry 1998;59(suppl 20):22-33.

Szentagotai A, David D: The efficacy of cognitivebehavioral therapy in bipolar disorder: a quantitative meta-analysis. J Clin Psychiatry 2010;71:66-72.

Vasak G, Katschnig H: Sturzfliegen: Leben in Depressionen und Manien. Zürich, Rüffer \& Rub Sachbuchverlag, 2001.

Wagner P, Bräunig P: Psychoedukation bei bipolaren Störungen. Ein Therapiemanual für Gruppen. Stuttgart, Schattauer, 2004 